\title{
Gene polymorphism IL13 in moderate-to-severe asthmatic Siberian children with different diseases control
}

\author{
Marina Smolnikova \\ Laboratory of cell-molecular \\ physiology and pathology \\ Scientific Research Institute of Medical \\ Problems of the North FRC KSC SB \\ RAS \\ Krasnoyarsk, Russia \\ smarinv@yandex.ru \\ Sergey Tereshchenko \\ Clinical department somatic and \\ mental health of children \\ Scientific Research Institute of Medical \\ Problems of the North FRC KSC SB \\ RAS \\ Krasnoyarsk, Russia \\ legise@mail.ru
}

\author{
Nina Gorbacheva \\ Clinical department somatic and \\ mental health of children \\ Scientific Research Institute of Medical \\ Problems of the North FRC KSC SB \\ RAS \\ Krasnoyarsk, Russia \\ n-n-gorbacheva@yandex.ru
}

\author{
Marina Malinchik \\ Laboratory of cell-molecular \\ physiology and pathology \\ Scientific Research Institute of Medical \\ Problems of the North FRC KSC SB \\ RAS \\ Krasnoyarsk, Russia \\ seapear11995@gmail.com
}

\begin{abstract}
Multifunctional cytokines, such as IL-13, play an important role in asthma pathogenesis. Several single nucleotide polymorphisms of cytokines are associated with asthma susceptibility in specific populations; however, further replicative studies in other ethnic groups are mandatory, especially with respect to asthma severity in children. Child with moderate-to-severe asthma were divided to patients uncontrolled course of the disease $(n=107)$ and controlled course of the disease $(n=95)$. The frequency of the $\mathrm{CT}$ genotype IL13 (rs1800925) in patients with controlled moderate-to-severe asthma was significantly higher in comparison with control $(46,2 \%$ / 36,6\%, OR 1,51, $p=0,03)$. Was obtained statistically significant differences in the frequency of the TT genotype between the population sample $(6,7 \%)$ and the group with uncontrolled asthma $(15,5 \%$, OR $1,65, p=0,03)$. The results have contributed to the data on the role of polymorphisms of IL-13 into the development of asthma in children as exemplified by a European population of East Siberia, Russia.
\end{abstract}

Keywords - bronchial asthma, IL13, gene polymorphism, disease severity

\section{Introduction}

Bronchial asthma (BA) is a multifactorial disease, since both environmental factors and the person's genetic predisposition contribute to the development of the disease [1, 6]. A number of associative relationships of cytokine gene polymorphism produced by Th2 cells with the development of bronchial asthma have been obtained [1]. Interleukin-13 (IL13 ) is the central mediator involved in the pathogenesis of bronchial asthma. In the bronchial tissues in asthmatic patients, there is a cell expansion expressing IL-13 mRNA, with overexpression of IL-13 in the lungs accompanied by the development of allergic inflammation, mucus hypersecretion, airways hyperresponsiveness, subepithelial fibrosis and eotaxin production [2]. Currently, anti-IL-13 (the trade name is "Lebrikizumab") is used in the treatment of bronchial asthma, which is a monoclonal antibody that blocks the activity of IL-13. When applying Lebrikizumab in patients, a significant improvement in the volume of forced expiratory flow for the first second of maneuver (FEV1) was observed [3].
The IL13 gene has a number of polymorphisms associated with the clinical manifestations of BA. Of particular interest is the study of rs 1800925 polymorphism due to its location in the promoter region of the IL13 gene, as well as the revealed association between this SNP and BA in both adult and children populations [4]. Carriers of the homozygous variant for the minor allele of this polymorphism developed an association with increased IL-13 production, airway hyperreactivity, and a positive skin test for allergen, and heterozygotes and homozygotes for the minor allele are sure to be associated with an elevated concentration of $\operatorname{IgE}[4,5]$.

The purpose of the study was to evaluate the associations between rs1800925 polymorphism of the IL13 gene and bronchial asthma with different control levels and disease severity in children of Caucasian origin in Eastern Siberia.

\section{Matirials and methods}

In the studied group of people there were children with bronchial asthma $(n=202$, average age was $12.8 \pm 1.2$ years old) and practically healthy children and adults $(n=135)$ in Krasnoyarsk. The control group included children $(n=33$, average age was $13.6 \pm 2.5$ years old) and adults with no history of BA and allergies $(\mathrm{n}=102$, average age $38.3 \pm 5.4$ years). A comparative analysis of the allele frequency in the control groups of different ages was carried out, no statistically significant differences were found, which allowed one to combine individuals of different ages in one control group. Child patients were divided into groups: moderate-tosevere BA with an uncontrolled course of the disease (UBA, $\mathrm{n}=107)$ and moderate BA with a controlled course of the disease $(\mathrm{CBA}, \mathrm{n}=95)$. The diagnosis, severity and the disease progression control was established upon recommendation of the GINA-2018.

DNA from peripheral blood was isolated using salting out method. Genotyping of IL13 (rs1800925) was carried out using the RT-PCR. Comparison of the allele frequency and genotypes between groups was provided using an online calculator, $\chi$-square test (http://gen-exp.ru/calculator_or.php). 


\section{Results and discussion}

The frequency of the alleles rs 1800925 of the IL13 gene in the control group obtained during the study corresponds the distribution in Caucasoid population: the frequency of the $\mathrm{C} *$ allele is $75 \%$, the $\mathrm{T} *$ allele is $25 \%$ (according to http://www.ensembl.org).

A statistically significant difference in the frequency of genotypes between BA patients and the control group was shown by comparison of the rs1800925 genotype frequency. Genotypes with the $\mathrm{T}^{*}$ allele are more common in patients as compared to control (OR $1.53(1.08-2.15), \mathrm{p}=0.027)$, which might indicate that $\mathrm{CT}$ and $\mathrm{TT}$ genotypes are risk factors for BA development. The frequency of the CT genotype in patients with UBA is statistically significantly higher than in the control group $(46.9 \%$ and $46.6 \%$ vs. $36.3 \%, p=0.008$, $\mathrm{p}=0.033$, respectively). This genotype can be assumed to be associated with UBA. Significant differences comparing the TT genotype frequency between the control group and the group with severe BA was also obtained. Thus, in the severe BA group, the homozygous TT genotype is more common $(15.49 \%$ vs. $6.7 \%, p=0.027)$. Therefore, this genotype is associated with the progression of pathology, i.e. the development of severe bronchial asthma.

In general, our results are consistent with previous studies on the association of bronchial asthma with the rs 1800925 polymorphism of the IL13 gene. According to Liu Z. et al., an association was defined between the rs1800925 IL13 polymorphism and the risk of asthma in children, since the CT and TT genotypes compared to the CC genotype, as well as high IL-13 expression in serum, were more common in the patient group. Using the further stratification of the study on ethnicity, a higher risk of the BA development in Caucasians compared to Mongoloids was shown [6]. Meta-analysis performed by Yang H.et al., revealed that rs1800925 polymorphism is associated with the risk of BA development among Caucasians [7].

\section{CONCLUSION}

Thus, Th2-lymphocytes play an important role in the pathogenesis of bronchial asthma, and some single-nucleotide variants of the IL13 gene are associated with a risk of the development, course and progression of bronchial asthma in children was confirmed by the obtained data.

\section{REFERENCES}

[1] S. Georas, J. Gue, U. Fanis, V. Casolaro, "T-helper cell type-2 regulation in allergic disease", European respiratory journal, 2005, vol. 26, pp. 933-947.

[2] X.X. Yang, F.X. Li, Y.S. Wu, D. Wu, J.Y. Tan, M. Li, “Association of TGF-beta1, IL-4 and IL-13 gene polymerphisms with asthma in a Chinese population", Asian Pacific Journal of Allergy and Immunology, 2011, vol. 29(3), pp. 273-277.

[3] J. Corren, "Role of interleukin-13 in asthma. Curr Allergy Asthma Rep.”, 2013, vol. 13(5), pp. 415-420.

[4] L. Cui, J. Jia, C.F. Ma, S.Y. Li, Y.P. Wang, X.M. Guo, Q. Li, H.B. Yu, W.H. Liu, L.B. Gao, "IL-13 polymorphisms contribute to the risk of asthma: a meta-analysis", Clinical Biochemistry, 2012, vol. 45, pp. 285-288.

[5] B. Beghe, I.P. Hall, S.G. Parker, M.F. Moffatt, A. Wardlaw, M.J. Connolly, L.M. Fabbri, C. Ruse, I. Sayers, "Polymorphisms in IL13 pathway genes in asthma and chronic obstructive pulmonary disease", Allergy, 2010, vol. 65(4), pp. 474-481.

[6] Z. Liu, P. Li, J. Wang, Q. Fan, P. Yan, X. Zhang, B. Han, "A metaanalysis of IL-13 polymorphisms and pediatric asthma risk", Medical Science Monitor: International Medical Journal of Experimental and Clinical Research, 2014, vol. 11(20), pp. 2617-2623.

[7] H. Yang, H. Dong, Y. Dai, Y. Zheng, "Association of interleukin-13 C-1112T and G+2044A polymorphisms with asthma: a meta-analysis", Respirology, 2011, vol. 16(7), pp. 1127-1135. 